
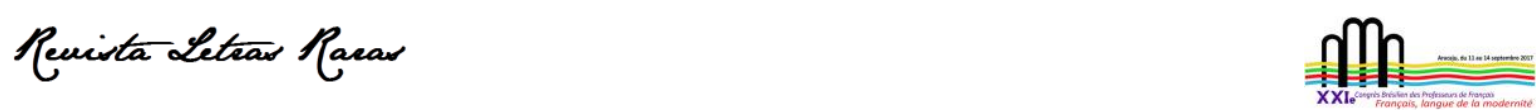

Actes du XXlème Congrès Brésilien des Professeurs De Français dans Édition spéciale de la Revue Letras Raras, 2021

ISSN : 2317-2347 - v. 10, Dossier Spécial (2021)

Todo o conteúdo da RLR está licenciado sob Creative Commons Atribuição 4.0 Internacional

\title{
L'enseignement des langues en quête d'une perspective sur
}

\section{l'égalité de chances}

\section{Christianne Benatti Rochebois*}

Licence en Lettres (Universidade Federal de Minhas Gerais - 1993). DEA en Sciences du langage, de l'homme et de la société (Université de Franche-Comté - 2003). Doctorat en Didactique des langues et des cultures (Université Paris III Sorbonne-Nouvelle - 2010). Professeur à Universidade Federal do Sul da Bahia.

(iD) https://orcid.org/0000-0002-5905-6493

Reçu en : 21 mai 2021. Approuvé en: 12 juillet 2021.

\section{Comment citer cet article:}

ROCHEBOIS, Christianne Benatti. L'enseignement des langues en quête d'une perspective sur l'égalité de chances. Revista Letras Raras, p. 269-278, n. Spécial, v. 10, nov. 2021.

\section{RÉSUMÉ}

La notion d'inclusion dans l'éducation s'est élargie au cours du temps vers le nouvel idéal de l'ouverture de l'environnement à l'autre et à son adhésion sociale. L'usage du terme suscite des débats, des controverses, pour ses définitions - et leurs élargissements - ainsi que pour les conséquences qu'ils supposent dans les pratiques. Dans notre parcours nous présentons quelques formats de définition et de mise en pratique d'un processus qui travaille et oscille en fonction des conditions locales, des politiques éducatives et des capacités des acteurs de l'éducation. Ensuite, après la narrative d'exemples dans quelques pays, notamment le Brésil, le texte expose quelques propositions didactiques possibles autour de l'articulation entre éducation inclusive et l'enseignement/apprentissage du français.

MOTS-CLÉS : Éducation ; Inclusion ; Enseignement/apprentissage du français.

\section{RESUMO}

A noção de inclusão na educação se expande na linha do tempo para o novo ideal de abertura do meio-ambiente ao outro e a sua adesão social. O uso do termo provoca debates, controvérsias, para suas definições - e suas expansões - assim como para suas consequências previstas nas práticas. Em nosso percurso apresentamos alguns formatos de definição e da prática de um processo que trabalha e oscila em função das condições locais, das políticas educativas e das capacidades dos atores da educação. Em seguida, após a narrativa de alguns exemplos em alguns países, principalmente o Brasil, o texto expõe algumas propostas didáticas possiveis sobre a articulação entre educação inclusiva e o ensino/aprendizagem de francês.

PALAVRAS-CHAVE: Educação; Inclusão; Ensino/aprendizagem de francês.

$\triangle$ chrisrochebois@hotmail.fr 

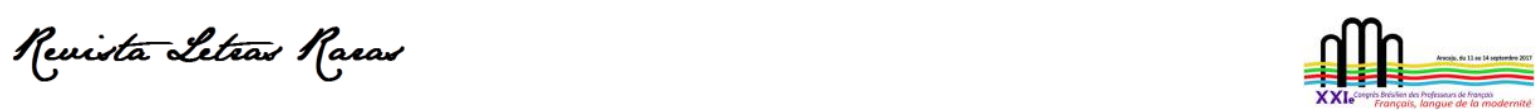

Actes du XXlème Congrès Brésilien des Professeurs De Français dans Édition spéciale de la Revue Letras Raras, 2021 ISSN : 2317-2347 - v. 10, Dossier Spécial (2021)

Todo o conteúdo da RLR está licenciado sob Creative Commons Atribuição 4.0 Internacional

\section{Introduction}

Qu'entendre par éducation inclusive? Comment se reconnaître dans l'extension des interprétations de cette notion préconisée par des organisations et mouvements dans le monde entier et qui est devenue l'essence de la politique de nombreux pays?

D’après Éric Plaisance, la notion d'inclusion a

... un caractère normatif selon les aspects suivants : son étymologie latine, ses usages initiaux dans les politiques internationales d'éducation et leur évolution vers la valorisation de la diversité ; ses variations de signification selon les pays en fonction de leurs politiques et de leurs institutions...; sa signification par rapport à deux notions : l'exclusion à laquelle elle s'oppose ; l'intégration dont elle se démarque ; ses conséquences paradoxales selon les analyses des sciences humaines et sociales contemporaines. (PLAISANCE, 2020, p.1)

Alors, il faut considérer la notion d'après sa présence dans les politiques éducatives, sur le plan international, national, voire régional. La notion d'inclusion dans l'éducation s'est élargie au cours du temps vers le nouvel idéal de l'ouverture de l'environnement à l'autre et à son vécu social. La question qui est à l'ordre du jour pour les chercheurs, les didacticiens et les praticiens est celle des contradictions et des ambiguïtés dans la mise en pratique.

Carlos Roberto Jamil Cury, dans sa définiton de l'éducation inclusive est opposé à l'idée de fermeture et valorise le droit à la scolarisation pour tous :

Dans son principe, l'éducation inclusive correspond à une modalité de scolarisation où ceux qui étudient ont les mêmes droits que leurs pairs, sans discrimination de sexe, de race, d'ethinie, de religion et de capacité. IIs disposent donc du droit de fréquenter les mêmes établissements et de participer aux activités des élèves de leur âge. (CURY, 2009, p. 42)

L'usage du terme éveille donc des débats, des controverses, pour ses définitions - et leurs élargissements - ainsi que pour les conséquences qu'ils supposent dans les pratiques. 

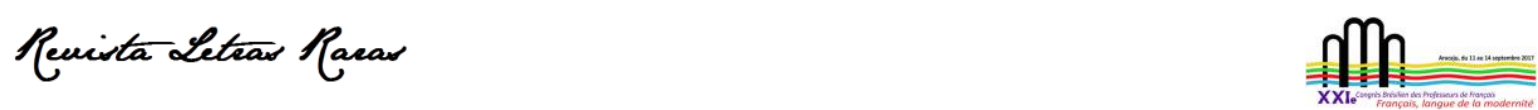

Actes du XXlème Congrès Brésilien des Professeurs De Français dans Édition spéciale de la Revue Letras Raras, 2021 ISSN : 2317-2347 - v. 10, Dossier Spécial (2021)

Todo o conteúdo da RLR está licenciado sob Creative Commons Atribuição 4.0 Internacional

Dans les documents les plus récents de l'UNESCO, la notion d'inclusion est au premier plan et le public prétendu est caractérisé par sa diversité, faisant référence à l'ensemble des apprenants et non à des types spécifiques: «L'éducation inclusive renforce la capacité du système éducatif à atteindre tous les apprenants et peut donc être comprise comme une stratégie pour réaliser l'éducation pour tous (EPT) [...] L'éducation est ainsi envisagée comme un processus qui vise à prendre en compte et à satisfaire la diversité des besoins de tous. » (UNESCO, 2009, p.8-9)

En 2020, l'Unesco a publié un rapport détaillé sur l'éducation et l'inclusion dans le sens que la diversité doit être vue comme une occasion propice aux changements de pratiques éducatives, au lieu d'être une difficulté. «Cependant, le rapport mentionne que, parmi les pays enquêtés, $68 \%$ fournissent bien une définition de l'éducation inclusive, mais $57 \%$ ne se réfèrent pas aux groupes sociaux marginalisés et $26 \%$ réduisent l'éducation inclusive aux handicaps et aux besoins spéciaux. » (PLAISANCE, 2020, p.3)

Nous allons dans le sens d'une approche universaliste pour le terme d'éducation inclusive, c'est-à-dire, qui considère l'adhésion du système scolaire à la différence en ce qui concerne l'accès, la réussite et l'insertion scolaire, ayant comme enjeu principal penser le besoin éducatif à partir de l'environnement éducatif qui entoure l'apprenant. Donc, c'est l'inclusion qui devient le mot-clé pour l'application du droit à la diversité, en définissant des changements importants dans les méthodologies éducatives, la formation des professeurs et les pratiques coopératives.

D'après l'expression de Pierre Bourdieu, des exclus de l'intérieur (BOURDIEU, 1992), nous touchons sur un point sensible dans la notion d'inclusion au Brésil et ailleurs. "Le placement à l'intérieur d'un même lieu ne signifie pas nécessairement la fin des situations de ségrégation. » (PLAISANCE, 2020, p.5). Les apprenants peuvent se trouver, ensemble, dans la même classe, dans la même école et subir des marginalisations quelquefois inaperçues et qui peuvent même exacerber l'exclusion sociale d'élèves issus de populations minoritaires.

Cela dit, nous nous trouvons en face d'une définition qui peut atteindre l'ensemble d'institutions, mais qui présente des variations et des niveaux, des cohérences et des paradoxes et qui, surtout, doit être considérée comme un processus qui travaille et oscille en fonction des conditions locales. 

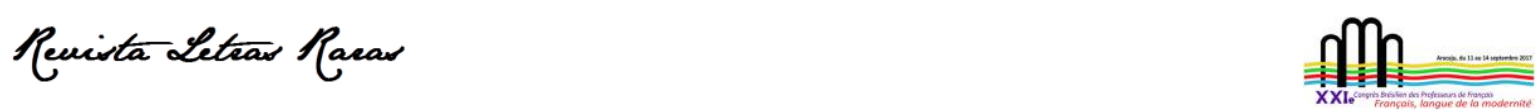

Actes du XXlème Congrès Brésilien des Professeurs De Français dans Édition spéciale de la Revue Letras Raras, 2021 ISSN : 2317-2347 - v. 10, Dossier Spécial (2021)

Todo o conteúdo da RLR está licenciado sob Creative Commons Atribuição 4.0 Internacional

Dans nos recherches et pratiques à l'université et dans des écoles, nous adoptons une définition de l'inclusion centrée dans le partage : des valeurs, des idées, des connaissances, des tâches éducatives entre différents partenaires dans et hors l'école ou des modalités de recherche; en tant que ressource pour la transformation des pratiques éducatives ouvertes à la diversité, mais à condition d'avoir des supports politiques et de son environnement.

Les recherches de terrain et leurs approches permettent de situer l'éducation inclusive à différents niveaux de complexité, soit chez les apprenants, soit chez les élèves.

Au Canada, pays très avancé vers l'éducation inclusive à tous niveaux, l'un des sites y destinés préconise les principes suivants de l'éducation inclusive :

« Les six principes suivants sont la clé de la réussite (...) en ce qui a trait à un système d'éducation inclusif. Ces principes peuvent guider et servir de base à la prise de décisions fondées sur les valeurs et centrées sur l'apprenant en ce qui a trait aux politiques, pratiques et mesures à tous les niveaux du système d'éducation.

1. Anticiper, valoriser et soutenir la diversité de chaque apprenant et les différences entre les apprenants.

2. Attentes élevées pour tous les apprenants.

3. Comprendre les points forts et les besoins des apprenants.

4. Réduire les obstacles au sein des environnements d'apprentissage.

5. Renforcement des capacités.

6. Responsabilité partagée. »

Disponible : [http://education.alberta.ca/éducation-inclusive/quest-ce-quelinclusION/]. Consulté : le 11 mai 2021.

Dans une société où chacun subit un flot d'informations, le rôle de l'école est moins d'imposer des contenus tout faits que de former à analyser ceux auxquels on est soumis et à savoir trier ce qui est pertinent ou ne l'est pas. Une telle démarche est en même temps une éducation à la citoyenneté, car elle implique d'écouter l'autre, de respecter ses idées et de résoudre les conflits par le discours et non par la force symbolique ou physique.

Plus spécifiquement, pour notre domaine de travail, l'enseignement/apprentissage du Français Langue Étrangère, si pour la majorité des Français leur langue agit comme un lien social, comme "source très importante de notre identité devant les valeurs de la République, de I'histoire, la culture et ses symboles" (Le Figaro, le 14 mars 2017), qu'en est-il des actions chez 

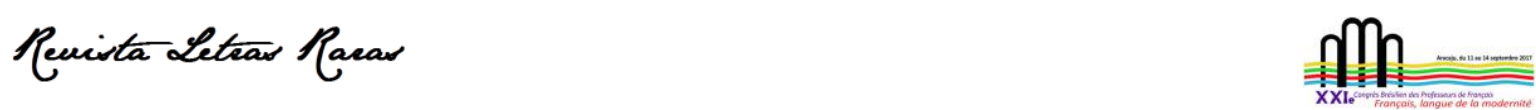

Actes du XXlème Congrès Brésilien des Professeurs De Français dans Édition spéciale de la Revue Letras Raras, 2021 ISSN : 2317-2347 - v. 10, Dossier Spécial (2021)

Todo o conteúdo da RLR está licenciado sob Creative Commons Atribuição 4.0 Internacional

nous, au Brésil, pour mettre à jour l'enseignement de la langue intégré avec ce mot devenu clé pour l'union des hommes et leurs territoires, l'éducation inclusive?

Nous exposons ensuite un exemple lu sur l'article «L'inclusion par l'éducation partagée » de Joanne Depeller et Danielle Zay, l'étude de cas d'une école sur le point de fermer en Espagne et qui a été transformée en mobilisant les ressources humaines existant dans la communauté environnante pour soutenir l'apprentissage. Des groupes interactifs ont été constitués avec des élèves hétérogènes par « le niveau de rendement dans le domaine, le genre, la culture, l'origine ethnique. Les adultes participants proviennent de milieux multiples, « étudiants, bénévoles de la communauté, membre des familles, anciens élèves et autres personnes du quartier » (DEPELLER et ZAY, 2015, p.1). La diversité des interactions devient un avantage qui aide à briser les stéréotypes sociaux. Des voisins se sont engagés dans la gestion en dehors de l'école, s'organisant pour la création et l'exécution des travaux. La conclusion est ressentie par les espaces de dialogue mixtes à l'école, formulant des solutions efficaces pour la communauté.

Cette expérience présente les avantages d'une politique éducative locale, qui s'appuie sur son environnement social. C'est les résultats d'une recherche sur une école primaire espagnole, mais sa portée va au-delà de l'étude de cas présentée puisque la transférabilité des résultats a été étudiée dans plusieurs centres éducatifs européens. (DEPPELER, 2017)

A Porto Seguro, au Bahia, l'Université du Sud de Bahia met en œuvre un projet d'intégration pédagogique dans une école publique de l'État. Sous la responsabilité d'une enseignante de l'université, une équipe analyse les difficultés rencontrées par un système éducatif déficient, et qui s'est toujours orienté par une politique gouvernementale d'éducation qui ne met pas en relief le territoire et ce qui vient avec.

En effet, de nombreux dysfonctionnements apparaissent, tant à cause de la distance entre les contenus offerts et le public, que des évaluations imposées par le système. Le projet de base intègre le personnel de l'école, de l'université et les étudiants de deux établissements. 

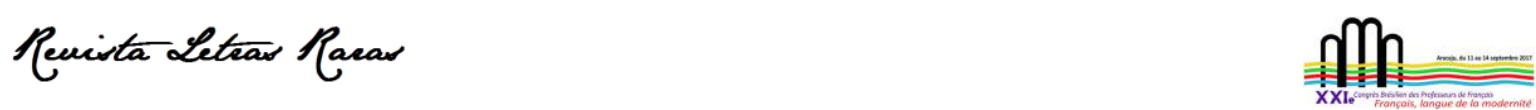

Actes du XXlème Congrès Brésilien des Professeurs De Français dans Édition spéciale de la Revue Letras Raras, 2021 ISSN : 2317-2347 - v. 10, Dossier Spécial (2021)

Todo o conteúdo da RLR está licenciado sob Creative Commons Atribuição 4.0 Internacional

L'effort est dirigé vers les intérêts culturels et professionnels de la communauté locale et régionale. Les programmes sont élaborés par tous les acteurs et les premiers effets, après quelques années de travail, attirent l'attention surtout par le changement de l'ambiance scolaire, la demande croissante des jeunes de Porto Seguro pour cette école, les résultats plus satisfaisants en matière du Bac, entre autres.

Les deux exemples, concernant une commune espagnole et une école publique bahianaise vont dans le sens de politiques d'éducation inclusive différentes, l'une fondée sur l'action locale, l'autre visant à se réinventer par une action intégrée pour centrer les pratiques dans les intérêts du territoire. Observons que, dans l'un et l'autre cas, la réalisation d'une telle politique s'appuie sur une conception similaire d'éducation partagée. Dans le premier, on préconise les liens avec les instances de l'environnement de l'école constituant le milieu dans lequel évolue l'apprenant. Dans le second, on situe l'initiative sur les liens entre les partenariats université-école pour soutenir un système éducatif plus inclusif dans son ensemble. Bref, toute démarche associant les plus d'acteurs sociaux concernés par l'éducation a des chances de trouver de meilleures solutions.

A la fin, un processus d'éducation inclusive implique non seulement les éducateurs, mais un dialogue entre ceux-ci, les autres citoyens et les représentants politiques pour aller dans le sens, à la fois, de réformes pédagogiques et de changements de société plus justes, les unes et les autres étant liés aux conceptions et aux valeurs de tous.

Depuis 2000, le Programme commun européen LINGUA exprime, comme première urgence, la mise en place de ce que l'on appelle, selon les pays, la pédagogie différenciée, l'enseignement ouvert, l'attention à la diversité des élèves ou encore l'apprentissage autonome. II ne s'agit pas de prendre prétexte de la différenciation pour valider des ségrégations mais d'ouvrir des espaces de négociation pour inciter l'apprenant à devenir acteur de ses apprentissages.

\section{Quelques propositions didactiques possibles autour de l'articulation entre éducation inclusive et l'apprentissage du français}

La pédagogie de l'inclusion connaît un large développement au Canada. Elle nous interroge au Brésil, car inclure par l'apprentissage des langues peut vouloir dire ouvrir des 

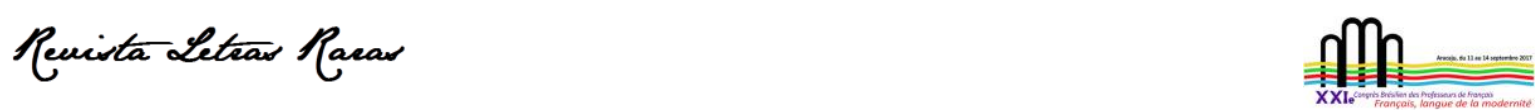

Actes du XXlème Congrès Brésilien des Professeurs De Français dans Édition spéciale de la Revue Letras Raras, 2021 ISSN : 2317-2347 - v. 10, Dossier Spécial (2021)

Todo o conteúdo da RLR está licenciado sob Creative Commons Atribuição 4.0 Internacional

espaces plurilingues. Cette pédagogie incite aussi à la coopération, à l'appui en même temps qu'à l'autonomisation. Inclure permet de créer lien, de partager son vécu et ses expériences. L'apprenant peut alors se sentir appartenir à sa société et au monde au-delà de son territoire (soit virtuel, soit réel).

On peut transmettre aux enseignants des informations sur les concepts d'interculturel, communautarisme, etc., ainsi que des éléments sociologiques et historiques sur l'éducation inclusive, afin de contextualiser le phénomène. Il est intéressant de se questionner sur l'apport de l'enseignement des langues, spécialement, dans notre cas, le français, à la communauté locale/régionale en termes linguistique et notamment, culturel.

Les communautés, territoires ou pays (le local, le régional ou le global) qui s'aventurent oui, parce que jusqu'à l'instant, c'est encore des expériences ponctuelles - à instaurer, au moins essayer l'éducation inclusive dans leurs institutions, font partie de politiques d'éducation différenciées. Soit fondée sur l'action locale, soit celle qui recherche d'adoucir les conséquences d'années des comportements méprisants de la diversité, ou l'autre qui expérimente avec tous et en tout pour changer les pratiques. Mais dans tous ces cas, les désirs et réalisations s'appuient sur une conception d'éducation partagée.

Pour l'ensemble des contextes et de démarches, nous nous trouvons accordés avec Danielle Zay :

...le processus d'éducation inclusive implique non seulement les éducateurs, mais un dialogue entre ceux-ci, les autres citoyens et les hommes politiques pour aller dans le sens, à la fois, de réformes pédagogiques et de changements de société plus justes, les unes et les autres étant liés aux conceptions et aux valeurs de tous. (DEPELLER et ZAY, 2015, p. 3)

Ensuite, nous présentons des projets pédagogiques solitaires, spécifiques et qui ont trouvé des résultats réellement importants dans leurs régions, pour essayer de démontrer autant leurs fragilités que leur réussite.

\section{Arts du langage}



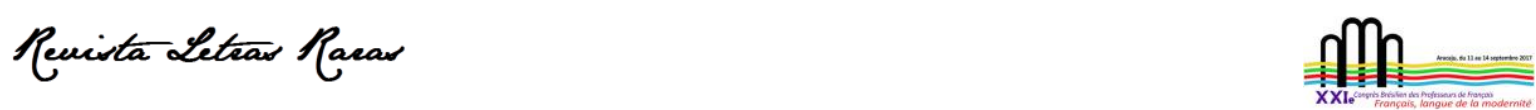

Actes du XXlème Congrès Brésilien des Professeurs De Français dans Édition spéciale de la Revue Letras Raras, 2021 ISSN : 2317-2347 - v. 10, Dossier Spécial (2021)

Todo o conteúdo da RLR está licenciado sob Creative Commons Atribuição 4.0 Internacional

Une entrée possible et travaillée à l'école publique de Porto Seguro est l'initiation au français par les pratiques artistiques (littérature, cinéma, théâtre, arts plastiques, musique...). La tension maintenue entre le technique et le créatif, le mimétique et le subjectif permet l'ancrage du jeu de la langue. Les arts du langage peuvent aider à devenir acteur dans une nouvelle langue, une nouvelle culture. Les échanges transversaux entre divers espaces théoriques et pratiques peuvent ouvrir de nouvelles voies pour explorer les relations entre arts et langages, là où le sujet, dans l'entre-deux des langues et des cultures, aura plus que jamais son mot à dire.

Les arts du langage semblent constituer un apport de premier ordre pour ces publics. L'apprenant prend des risques quand il s'expose aux autres, mais il acquiert aussi plus de confiance. Ces pratiques offrent également la possibilité d'entrer dans le langage sans se trouver démuni comme cela peut être le cas face à des pratiques scolaires qui peuvent être inconnues. Les pratiques artistiques, universelles et singulières, peuvent se révéler un début de solution pour une rencontre avec l'autre texte, l'autre langue, l'autre culture.

Ces expériences favorisent la construction d'un savoir être (construction de soi et de la relation aux autres), puisque les actes de paroles les plus essentiels - se présenter, discuter, argumenter, exprimer ses goûts, son opinion - sont majeurs pour les pratiques artistiques. Enfin, ces activités s'inscrivent sous le signe de la pédagogie différenciée comme pour comparons nos langues puisque les compétences des élèves sont très hétérogènes. Les pratiques artistiques et culturelles favorisent des réalisations langagières et concrètes, selon le niveau de chacun sans processus de dévalorisation, par le travail personnel et d'équipe. La démarche se veut donc constructive.

\section{En classe et en scène}

Un exemple encore au Brésil, En classe et en scène est constitué par un groupe d'étudiants - tous les cycles compris, et des professeurs de l'Université de Brasilia où l'enseignement de la langue française à travers la préparation et la présentation des pièces théâtrales, se justifie dans une perspective collective et de collaboration où, d'après Gloria Magalhães, la responsable des travaux du groupe, le dans le lieu où on vit se place conjointement avec qui on vit, comme importance fondamentale pour la construction du travail. 

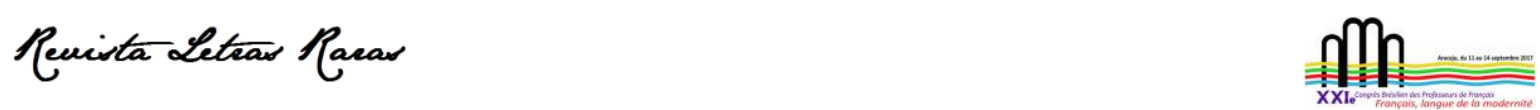

Actes du XXlème Congrès Brésilien des Professeurs De Français dans Édition spéciale de la Revue Letras Raras, 2021

ISSN : 2317-2347 - v. 10, Dossier Spécial (2021)

Todo o conteúdo da RLR está licenciado sob Creative Commons Atribuição 4.0 Internacional

C'est un faire théâtral dans un contexte d'éducation et de recherche, où la réflexion sur le rôle dans la société et dans l'université est discutée en permanence. La pratique part de I'hypothèse que la connaissance est conçue avec l'autre, dans un discours qui cherche l'écoute de la voix de toutes et tous, dès les participants du groupe au public. L'objectif est donner la voix aux apprenants - en français - et, à travers les relations des sujets mis côte à côte, dans une construction partagée et collective d'un processus où l'enseigner et l'apprendre sont dans un rapport dialectique et dialogique.

Pour conclure ce moment de réflexion, nous prenons la liberté de reproduire les mots de Maria da Gloria Magalhães dos Reis, dans sa présentation du groupe créé par elle-même, en respect aux personnes qui croient la formation comme un processus ouvert à maintes possibilités de lecture et de formation :

Dans notre expérience, nous prétendons offrir un espace où la littérature soit découverte à travers le plaisir du texte par moyen des pratiques qui passent par le corps et par la voix, en favorisant le développement de l'expression critique, proposant un théâtre de transformation. Travailler collectivement et en collaboration avec les étudiants et les personnes de nos communautés du District Fédéral, sur des textes en relation aux problèmes de notre société actuelle, par exemple, des guerres, de la violence, des attaques contre l'humanité en général, peut réveiller le désir de transformation, inciter à une volonté de changement en direction à une société dans laquelle l'altérité et la pluralité soient mises au premier plan. Dans les productions, lectures et recherches du groupe, nous apercevons le théâtre en tant qu'acte politique, dans une perspective où tout art est une action concrète dans le monde, sans, cependant, perdre de vue le travail artistique considéré dans sa forme expérimentale et son ambition philosophique. La tendance expérimentale du groupe, additionnée à la réflexion théorique, nous a amenés à la construction d'un théâtre politique, émancipateur et critique, en dialogue avec l'enseignement de langues et littératures. Un théâtre qui lance l'alerte sur les discours actuels de censure et d'oppression'. (REIS, 2021, p. 16, notre traduction).

\footnotetext{
${ }^{1}$ Em nossa experiência, pretendemos oferecer um ambiente em que a literatura seja descoberta por meio do prazer do texto por meio de práticas que passam pelo corpo e pela voz favorecendo o desenvolvimento da expressão crítica, se propondo como um teatro de transformação. Trabalhar coletivamente e em colaboração com estudantes e pessoas de nossas comunidades do Distrito Federal, sobre textos relacionados a problemas de nossa sociedade atual, como guerras, violência, ataques contra a humanidade em geral, pode despertar o desejo de transformação, incitar a uma vontade de mudança em direção a uma sociedade na qual a alteridade e a pluralidade sejam colocadas em primeiro plano. Nas produções, leituras e pesquisas do grupo, percebemos o teatro como um ato político, na perspectiva de que toda arte é uma ação concreta no mundo, sem, no entanto, perder de vista o trabalho artístico considerado em sua forma experimental e sua ambição filosófica. A tendência experimental do grupo, somada à reflexão teórica,
} 

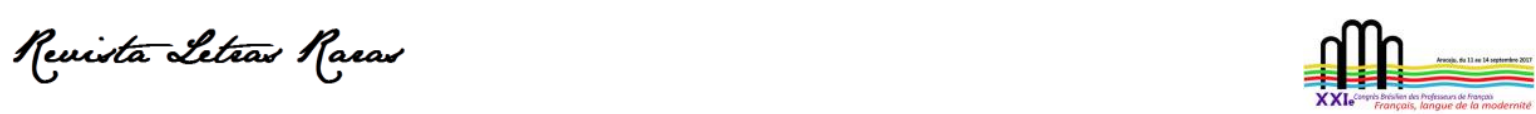

Actes du XXlème Congrès Brésilien des Professeurs De Français dans Édition spéciale de la Revue Letras Raras, 2021 ISSN : 2317-2347 - v. 10, Dossier Spécial (2021)

Todo o conteúdo da RLR está licenciado sob Creative Commons Atribuição 4.0 Internacional

\section{Références}

BOURDIEU, P, CHAMPAGNE, P. Les exclus de l'intérieur. Actes de la recherche en sciences sociales, n. 91-92, p. 71-74, 1992.

CURY, C. R. J. Réflexions sur les principes juridiques de l'éducation inclusive au Brésil. Égalité, droit à la différence, équité. Recherche et formation pour les professions de l'éducation. n. 61, p. 41-53, 2009.

DEPPELER, J., ZAY, D. L'inclusion par l'éducation partagée, Recherches et éducation - Revue généraliste de recherches en éducation et formation, En ligne: [https://rechercheseducations.revues.org/2351]. Consulté : le 5 août 2017.

PLAISANCE, É.. La reconfiguration du métier d'enseignant dans la dynamique de l'accessibilité. In: EBERSOLD, S. (org.). L'accessibilité ou l'école réinventée. London: ISTE/Wiley Editions, 2020.

REIS, M. da G. M. dos. En classe et en scène, Campinas : Pontes Editora, 2021.

UNESCO. Principes directeurs pour l'inclusion dans l'éducation. Paris: Unesco, 2009. En ligne: [https://inee.org/system/files/resources/UNESCO_Policy_Guidelines_on_Inclusion_in_Education_ 2009_FRE.pdf]. Consulté : le 8 août, 2017.

UNESCO. Promoting Inclusive Teacher Education. Advocacy Guides. Paris: Unesco, 2013. En ligne :

[https://en.unesco.org/inclusivepolicylab/sites/defaultfiles/learning/document/2017/1/221033e.pdf ]. Consulté : le 8 août, 2017.

UNESCO. Déclaration d'Incheon. Education 2030. Paris: Unesco, 2015. En ligne: [http://uis.unesco.org/sites/defaultffiles/documents/education-2030-incheon-framework-for-actionimplementation-of-sdg4-2016-fr.pdf]. Consulté : le 9 août 2017.

UNESCO. Inclusion and education: all means all. Global Education Monitoring Report. Paris: Unesco, 2020. En ligne: [https://en.unesco.org/gem-report/report/2020/inclusion]. Consulté : le 10 mai 2021.

levou-nos à construção de um teatro político, emancipatório e crítico, em diálogo com o ensino de línguas e literaturas. Um teatro de denúncia dos atuais discursos de censura e opressão. (REIS, 2021, p. 16) 